\title{
Effect of interdiffusion of quantum well infrared photodetector
}

\author{
Alex S. W. Lee and E. Herbert $\mathrm{Li}^{\mathrm{a}}$ \\ Department of Electrical and Electronic Engineering, University of Hong Kong, Pokfulam Road, \\ Hong Kong
}

(Received 14 August 1996; accepted for publication 7 October 1996)

\begin{abstract}
The intersubband infrared photodetector performance is theoretically analyzed for various stages of interdiffusion in $\mathrm{AlGaAs} / \mathrm{GaAs}$ quantum well. The absorption strength and responsivity are enhanced for certain extents of interdiffusion and the peak detection wavelength red shifts continuously with a large tunable range from 7 to $38.4 \mu \mathrm{m}$. The dark current is at an acceptable value for small diffusion extent. (C) 1996 American Institute of Physics. [S0003-6951(96)04749-3]
\end{abstract}

Since the first observation of intersubband absorption and a large dipole moment in III-V semiconductor quantum wells (QWs) were reported, ${ }^{1}$ there has been rapid progress in quantum well infrared (IR) photodetectors ${ }^{2-6}$ for the matured QW III-V material and device processing technologies. The principal operation parameters, such as quantized energy levels, are determined by the QW confinement profile and which depend on the dimension and alloy composition of the well and barrier. However, the designed (prior to growth) QW structure may not always turn out to be expected after growth owing to the inhomogeneities in well width, the nonconstant growth rate of the GaAs and AlAs, or the failure in precisely controlling the evaporation rate of $\mathrm{Ga}$ and $\mathrm{Al}$ during growth. These may lead to a lower yield rate since the electronic and optical properties of a QW requires a fairly precise accuracy. Interdiffusion provides a means to offer the flexibility to modify the properties of the material after growth. It has been demonstrated that the intersubband transition energy in a partially diffused quantum well (DFQW) can be red shifted ${ }^{7}$ and postgrowth tuning of the peak detection wavelength can be achieved in QW IR detectors using rapid thermal annealing. ${ }^{8}$ In this letter, we present the advantage of using the DFQW to modify the intersubband optical spectrum, such as absorption peak wavelength and spectral width. We will also demonstrate enhanced performance of the DFQW photodetector in absorption and responsivity with a wide tunable detection wavelength from 7 to $38.4 \mu \mathrm{m}$.

DFQW is formed by the interdiffusion of $\mathrm{Al}$ and $\mathrm{Ga}$ atoms across the well and barrier interface after a certain time and at a high enough temperature. The extent of interdiffusion is characterized by the diffusion length $L_{d}$ $=(D t)^{1 / 2}$, where $D$ is the diffusion coefficient and $t$ is diffusion time. The diffused $\mathrm{Al}$ composition profile, $w(z)$, across the QW structure is given by ${ }^{9}$

$$
w=w_{0}\left\{1-\frac{1}{2}-\left[\operatorname{erf}\left(\frac{L_{z}+2 z}{4 L_{d}}+\operatorname{erf}\left(\frac{L_{z}-2 z}{4 L_{d}}\right)\right]\right\},\right.
$$

where $w_{0}$ is the as-grown $\mathrm{Al}$ mole fraction in the barrier, $L_{z}$ is the grown axis (QW centered at $\left.z=0\right)$, and erf denotes the error function, $L_{d}$ is the diffusion length. The DFQW confinement profile for the conduction band, $U_{c}(z)$ modeled by error function, is defined by: $U_{c}(z)=Q_{c}\left[E_{g}(z)-E_{g}(z\right.$ $=0)$ ], where $Q_{c}=0.65$ is the band offset ratio and $E_{g}(w)$ is

${ }^{\text {a)} E l e c t r o n i c ~ m a i l: ~ e h l i @ h k u e e e . h k u . h k ~}$ the bulk band gap at room temperature. The other material parameters are adapted from Ref. 9. Using the Ben-Daniel and Duke model with a $z$-position dependent effective mass on the interdiffused composition profile, the energies of these states $\left(E_{c 1}, E_{c 2}, \ldots\right)$ can be solved by the Schrödinger equation using a finite difference method.

The DFQW structures in this study consist of 50 periods of $40 \AA$ wide as-grown QW alternating with $500 \AA$ $\mathrm{Al}_{0.3} \mathrm{Ga}_{0.7} \mathrm{As}$ barrier layers. The well is $n$-type doped with $1 \times 10^{18} \mathrm{~cm}^{-3} \mathrm{Si}$ and the material are interdiffused with different diffusion extents. The model of intersubband-band absorption in the QWs is based on the one-electron density matrix formulation ${ }^{10}$ with $P$-polarized incident photon, i.e., the electric field vector $\hat{\mathbf{E}}$ of the incident optical wave is oriented along the QW confinement direction ( $z$ axis) and takes into account the intrasubband relaxation. The absorption coefficient, $\alpha$, is given by

$$
\begin{aligned}
\alpha= & \frac{\omega}{c_{0} n_{r} \epsilon_{0}}\left|M_{f i}\right|^{2}\left(\frac{m^{*} k_{B} T}{L_{z} \pi \hbar^{2}}\right) \\
& \times \ln \left(\frac{1+\exp \left(\frac{E_{F}-E_{i}}{k_{B} T}\right.}{1+\exp \left(\frac{E_{F}-E_{f}}{k_{B} T}\right)}\right) \\
& \times \frac{\frac{\hbar}{\tau_{\text {if }}}}{\left(E_{f}-E_{i}-\hbar \omega\right)^{2}+\left(\frac{\hbar}{\tau_{\text {if }}}\right)^{2}},
\end{aligned}
$$

where $\omega$ is the photon frequency, $\mu$ is the permeability of the material, $c_{0}$ is the light velocity, $L_{z}$ is the well width, $m^{*}$ is the effective mass of electron, $\epsilon_{0}$ is the permittivity in vacuum, $\left|M_{\text {if }}\right|^{2}$ is the dipole matrix elements, $n_{r}=3.2$ is the refractive index and $\tau_{\text {if }}=90$ fs is the intrasubband relaxation time. ${ }^{11}$ The responsivity, $\mathfrak{R}$, is defined as the ratio between the output signal and the radiant input and is given by ${ }^{3}$

$$
\mathfrak{R}(v)=\frac{e}{h \nu} g \eta(v) P_{e},
$$

where $\eta(\nu)=1 / 2\left(1-e^{-2 \alpha(\nu) L_{p}}\right)$ is the quantum efficiency and the factor 2 accounts for the increased absorption due to the reflection off the top metallic contact, ${ }^{2} \alpha$ is the absorption coefficient, $g=L / l$ is the optical gain where $L$ is the hotelectron mean free path, and $l$ is the device length and $P_{e}$ 


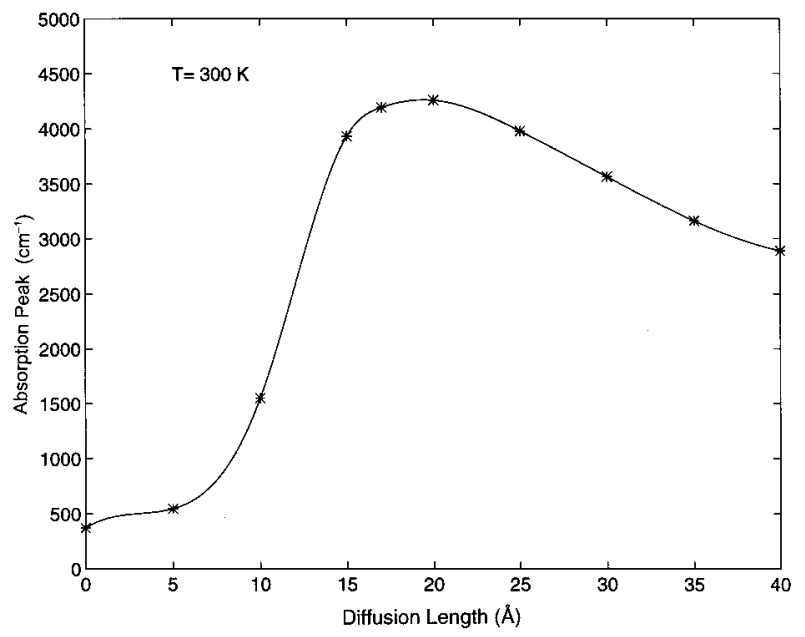

FIG. 1. Room-temperature absorption peak for $L_{d}=0-40 \AA$ of $\mathrm{Al}_{x} \mathrm{Ga}_{1-x}$ As $/$ GaAs DFQW $(x=0.3$, well width $=40 \AA)$.

$=\left(1+\tau_{e} / \tau_{r}\right)^{-1}$ is the tunneling escape probability ${ }^{5}$ where $\tau_{e}$ is the escape time from the vicinity of the well and $\tau_{r}$ is the recapture time. The factors $g=0.5$ and $P_{e}=0.8$ are taken for bound-to-continuum transition and $g=0.3$ and $P_{e}=0.5$ for bound-to-bound transition for simplicity of calculation. The dark current is calculated by ${ }^{4}$

$$
I_{D}(v)=A n_{t}(v) e \nu_{d}(v),
$$

where $A$ is the detector area, $e$ is the electronic charge, $\nu_{d}(v)=\mu F\left[1+\left(\mu F / \nu_{s}\right)\right]^{-1 / 2}$ is the conduction band drift velocity, where $F$ is the average field, $\nu_{s}=5 \times 10^{-6}$ is the saturation drift velocity and $\mu=1200 \mathrm{~cm}^{2} / \mathrm{V}$ s is the mobility. $n_{t}$ is the carrier density laying above the barrier conduction band edge and is given by

$$
n_{t}=\frac{1}{L_{p}} \int_{E 0}^{\infty} T(E, V) f(E) \rho(E) d E,
$$

where $L_{p}$ is the DFQW period, $V$ is the potential drop across a period, $E$ is the total energy, $E_{0}$ is the ground state energy, $f(E)=\left[1+\exp \left(E-E_{0}-E_{F}\right) k T\right]^{-1}$ is the Fermi factor, $E_{F}$ is the two-dimensional Fermi level, $\rho(E)$ is the twodimensional density of state in the $\mathrm{QW}$, and $T(E, V)$ is the bias-dependent tunneling probability through a single barrier and was calculated using the WKB method. In our calculation, $T(E, V)=1$ is taken for $E>V_{0}$, where $V_{0}$ is the barrier height and $T(E, V)=\exp \left\{\left[-4 L_{p} /(3 e V)\right]\left(2 m^{*} / \hbar^{2}\right)^{1 / 2}\left(V_{0}\right.\right.$ $\left.-E)^{3 / 2}-\beta\left(V_{c}-E-e V\right)^{3 / 2}\right\}$, where $\beta=0$ for $V_{0}-e V<E$ $<V_{0}$ and $\beta=1$ for $E_{0}<E<V_{0}-e V$.

Figure 1 shows the room-temperature intersubband transition absorption peak, $\alpha_{p}$, for several $L_{d}$ ranging from $L_{d}$ $=0$ to $L_{d}=40 \AA$. It can be seen that the absorption peak increases from $\alpha_{p}=369 \mathrm{~cm}^{-1}\left(L_{d}=0\right.$, i.e., an as-grown square QW where the first excited state is in the continuum) to $\alpha_{p}=4297 \mathrm{~cm}^{-1}\left(L_{d}=20 \AA\right)$, and then followed by a gradual monotonic decrease. The large enhancement in absorption strength is due to the fact that as interdiffusion proceeds, the intermixing of $\mathrm{Al}$ and $\mathrm{Ga}$ will result in a graded well shape where the ground state energy, first excited state energy, and the barrier height will be modified accordingly as shown in Fig. 2. For $L_{d}=20 \AA$, the first excited state is

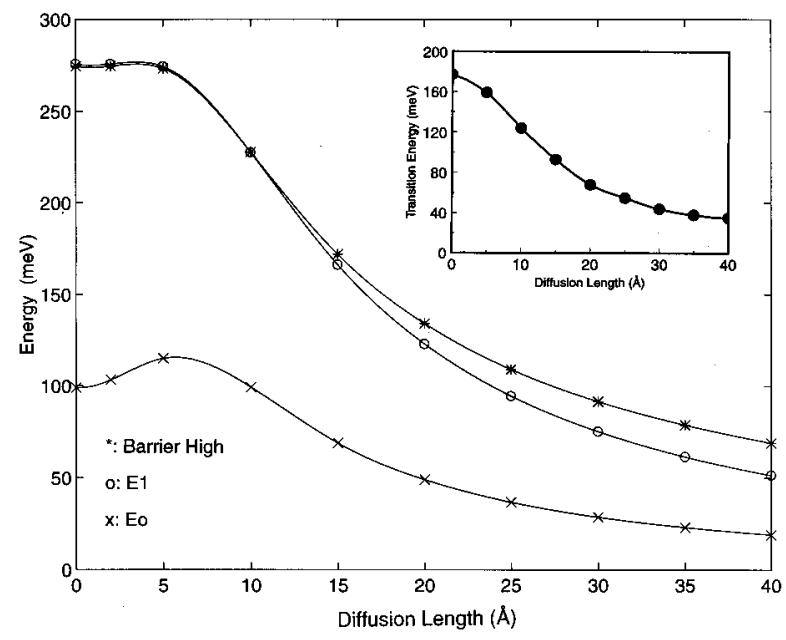

FIG. 2. Variation of energy levels for $L_{d}=0-40 \AA$. Inset is the transition energy for different $L_{d}$.

localized and bound in the DFQW at about $11 \mathrm{meV}$ below the top of the well, whose envelope wave function strongly overlaps with the ground state and results in a larger oscillator strength. This is consistent with the theory that the largest oscillator strength occurs when the first excited state is close to the top of the well ${ }^{12,13}$ which leads to an improved absorption strength. It is interesting to note that even though the ground state energy increases with $L_{d}(<7 \AA)$, the intersubband transition energy shows no abrupt change but monotonic red shifts, as shown in the inset of Fig. 2. This phenomenon agrees well with the experimental data ${ }^{7}$ although details of the ground stated variation has not been shown there. For a larger interdiffusion extent $\left(L_{d}\right.$ $>25 \AA$ ), the DFQW begins to "flatten" out with a wider effective well width and a lower barrier height, where the wave function extends far into the barrier. This reduces the quantum confinement effect and consequently a high probability for charges transfer out of the well, which leads to a reduced absorption strength.

Figure 3 shows the calculated responsivity spectra of a $10 \mathrm{kV} / \mathrm{cm}$ electric field at $77 \mathrm{~K}$ for different $L_{d}$. It can be seen that the responsivity spectrum increases in amplitude

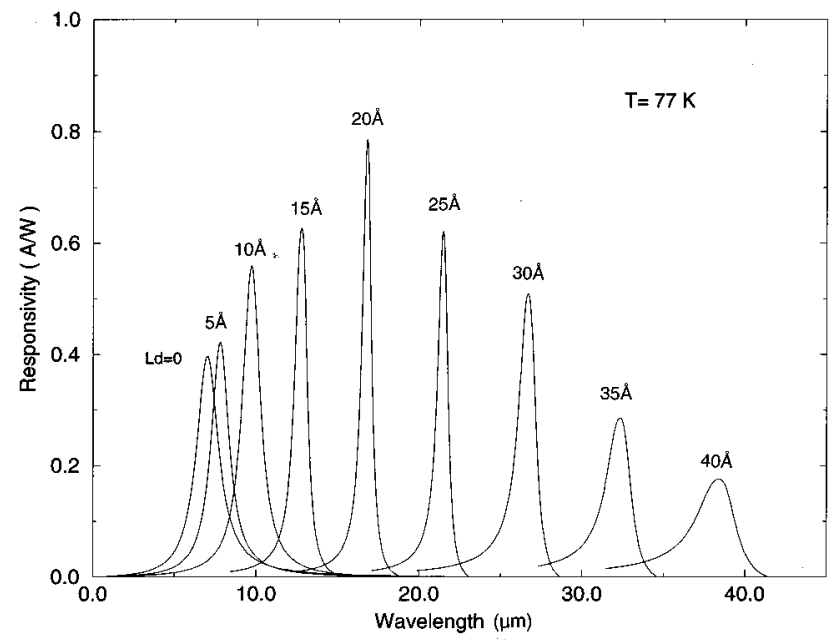

FIG. 3. Responsivity spectra at $T=77 \mathrm{~K}$ for $L_{d}=0-40 \AA$. 


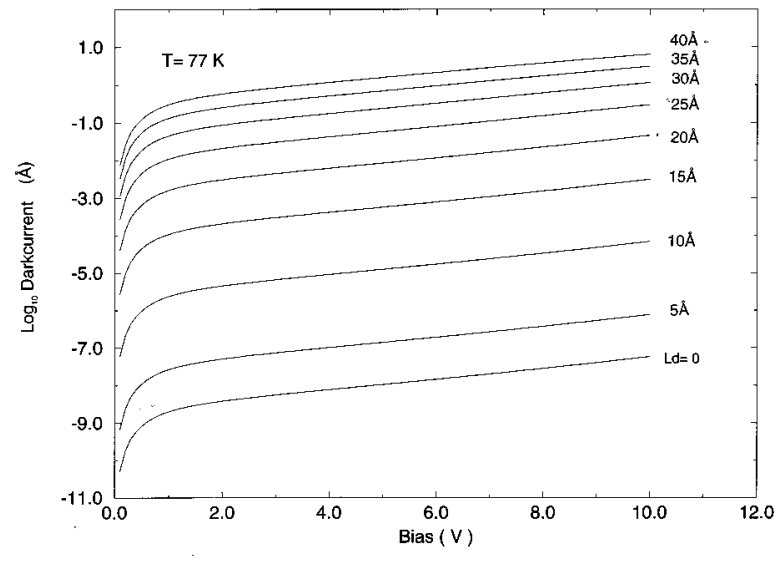

FIG. 4. Dark current vs bias at $T=77 \mathrm{~K}$ for $L_{d}=0-40 \AA$.

and narrows in linewidth as $L_{d}$ increases to $20 \AA$ A. As interdiffusion proceeds further, it reduces in amplitude while the linewidth becomes broader again. The peak responsivity red shifted throughout the range of interdiffusion considered here. Note that the response spectra remain broader for $L_{d}$ smaller than $10 \AA$. This is due to the bound-to-continuum transition where the first excited state remains above or at almost the same height as the barrier, and its wave function spreads over the continuum. For $L_{d}$ larger than $10 \AA$, the first excited state becomes more localized and bound within the DFQW. The transition is now bound-to-bound which leads to a larger oscillator strength and narrower linewidth as shown in Fig. 3. The broadening of the spectra at even larger $L_{d}$ is due to the flattening of the well shape, thus gradually losing its quantum confinement. With an applied field, the barrier height will be further reduced and this makes the DFQW even more flattened and bulklike in nature, and which leads to a smaller oscillator strength. The lowering of the barrier height reduces the tunneling time of the photoexcited electron out of the well which contributes to the broadening effect. ${ }^{3}$ Since the time constant in our model is fixed, we exclude this mechanism.

Dark current is analyzed here for different $L_{d}$ at $T=77$ $\mathrm{K}$, as shown in Fig. 4. The dark current is increased by about eight orders as $L_{d}$ increases from 0 to $40 \AA$ at a bias of $3 \mathrm{~V}$. However, the rate of increase of dark current decreases with increasing $L_{d}$. This is not surprising because the barrier height decreases with increasing $L_{d}$, as shown in Fig. 2 . Since tunneling through the top of the barrier is the major contribution to the dark current, the lowering of the barrier will assist the nonphotoexcited electron to tunnel out of the well. This agrees well with experimental data reported ${ }^{6}$ by varying both the well and barrier thickness of square QWs. Note that the dark current of $L_{d}=0$ and $5 \AA$ does not overlap but differs by an order even though they are of the same barrier height. This is owing to the increase of ground state energy which is due to a narrower effective well width of the DFQW profile during the initial stage of interdiffusion, as shown in Fig. 2. The dark current increase linearly for bias $>1 \mathrm{~V}$. Since the bottom of the square well will be changed gradually into a graded shape by interdiffusion, ${ }^{9}$ this will add to the barrier effectively an additional thickness to impede the tunneling of electrons so that the dark current increases with a smaller gradient.

In summary, we have reported a wide tuning range and an enhanced performance DFQW photodetector at various $L_{d}$. Absorption strength as well as responsivity can be enhanced and the detection wavelength can be tuned by modifying the energy levels of the QW through interdiffusion. Dark current increases by a few orders with interdiffusion due to the lowering of the barrier height, but for $L_{d}<15 \AA$ and small bias, the dark current is at an acceptable level for infrared detection applications. Optimizing the design of the DFQW will be able to reduce the dark current at larger $L_{d}$.

The authors would like to thank the HKU-CRCG and RGC-Earmarked Research Grant for financial support.

${ }^{1}$ L. C. West and S. J. English, Appl. Phys. Lett. 46, 1156 (1985).

${ }^{2}$ B. F. Levine, K. K. Choi, C. G. Bethea, J. Walker, and R. J. Malik, Appl. Phys. Lett. 51, 934 (1987).

${ }^{3}$ B. F. Levine, C. G. Bethea, K. K. Choi, J. Walker, and R. J. Malik, Appl. Phys. Lett. 53, 231 (1988).

${ }^{4}$ B. F. Levine, C. G. Bethea, G. Hasnain, V. O. Shen, E. Pelve, R. R. Abbott, and S. J. Hsieh, Appl. Phys. Lett. 56, 851 (1990).

${ }^{5}$ H. C. Liu, A. G. Steel, M. Buchanan, and Z. R. Wasilewski, in Intersubband Transitions in Quantum Wells, edited by E. Rosencher, B. Vinter, and B. Levine (Plenum, New York, 1992), p. 57.

${ }^{6}$ B. F. Levine, A. Zussman, S. D. Gunapala, M. T. Asom, J. M. Kuo, and W. S. Hobson, J. Appl. Phys. 72, 4429 (1992).

${ }^{7}$ J. D. Ralston, M. Ramsteiner, B. Dischler, G. Brandt, P. Koidl, and D. J. As, J. Appl. Phys. 70, 2195 (1991).

${ }^{8}$ A. G. Steele, M. Buchanan, H. C. Liu, and Z. R. Wasilewski, J. Appl. Phys. 75, 8234 (1994).

${ }^{9}$ E. H. Li, B. L. Weiss, and K. S. Chan, Phys. Rev. B 46, 15180 (1992).

${ }^{10}$ D. Ahm andS. L. Chuang, IEEE J. Quantum Electron. 23, 2196 (1987).

${ }^{11}$ R. Ferreira and G. Bastard, Phys. Rev. B 40, 1074 (1989).

${ }^{12}$ D. D. Coon and R. P. G. Karunasiri, Appl. Phys. Lett. 45, 649 (1984).

${ }^{13}$ G. Steele, H. C. Liu, M. Buchanan, and Z. R. Wasilewski, Appl. Phys. Lett. 59, 3625 (1991). 